Gibran Yusuf

\title{
Joint Winner of Young Investigator Award Euroson 2015
}

\section{Abstract \\ $\nabla$}

Multi-Parametric Ultrasonography of Testicular Haematomas: Features on Grey Scale, Colour Doppler, Contrast Enhanced Sonography and Strain Elastography

Gibran T Yusuf, Eleni Konstantatou, Maria E Sellars, Dean Y Huang, Paul S Sidhu King's College Hospital, London, UK

\section{Purpose \\ $\nabla$}

Testicular trauma results in a variety of testicular complications including an intra-testicular haematoma. A focal intratesticular lesion following trauma may be diagnostically difficult with a primary germ cell tumour a possibility. We investigate the appearances of a series of intratesticular haematomas in comparison to tumours on multi-parametric ultrasonography (MPUS).

\section{Methods \\ $\nabla$}

Hematomas/tumours were identified from a database and retrospectively reviewed by experienced observers. All patients underwent testicular ultrasonography (US), by experienced observers, using high frequency transducers, employing standard techniques of MPUS combining grey-scale, colour Doppler, contrast enhanced ultrasound (CEUS) and strain elastography (SE). Features recorded were; size, location, echogenicity, colour Doppler signal, contrast-enhancement, elasticity score and strain ratio. Follow-up was recorded.

\section{Results \\ $\nabla$}

17 haematomas (right testis $n=11$, left testis $\mathrm{n}=6$, maximum diameter range $5-28 \mathrm{~mm}$ ) in 8 patients (mean age 31 years, range 25-43 years) were analysed. Blunt trauma $(n=7)$ and surgery $(n=1)$ were preceding events. Greyscale sonography demonstrated well-defined, oval or round, predominantly hypoechoic and heterogeneous, peripherally $(n=13)$ or centrally located $(n=4)$. Three demonstrated CEUS enhancement of septations not seen on colour Doppler and 4 peripheral rim enhancement. Sixteen lesions had SE performed; mean strain ratio was 1.49 (range 0.41-2.77), elasticity score $\leq 3$ $(n=12)$ and $5(n=4)$. All lesions were confirmed benign on follow-up sonography. Retrospective analysis with similar size histologically proven tumours was performed (seminoma, Leydig cell tumours and non seminomatous germ cell tumours $\mathrm{n}=8, \mathrm{n}=5, \mathrm{n}=4$ respectively). Demographics were similar in both groups (mean age 37 years, range 25-64). Grayscale imaging demonstrated predominantly subcapsular peripheral lesions which were predominantly hypoechoic or heterogeneous $(n=16)$. The background testis was abnormal in $(n=6)$ with microlithiasis the most common findings. On CEUS all lesions definitively showed enhancement and were found to be subjectively hard on $\mathrm{SE}$ (mean strain ratio 6.04, range 0.61-15).

\section{Conclusion \\ $\nabla$}

The presence of intralesional vascularity can be definitively determined by CEUS and with SE demonstrating a "hard" lesion

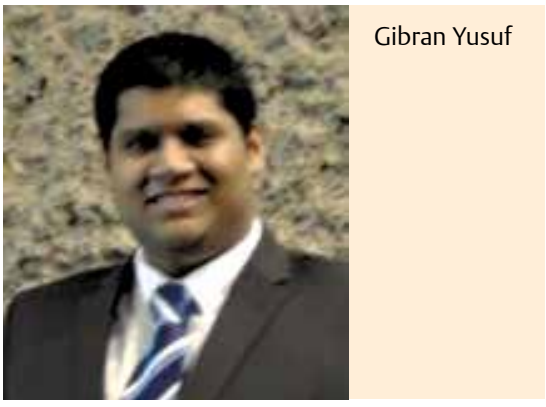

is a hallmark of tumours. Testicular haematomas are variable on SE with absence intralesional vascularity and resolution on follow up imaging confirmatory. MPUS aids the interpretation of the benign nature of intra-testicular haematoma allowing conservative management.

\section{Clinical Relevance}

$\nabla$

Testicular haematomas may mimic malignancy, particularly if a history of trauma is not forthcoming. The advent of CEUS and SE can increase the certainty of the diagnosis and avoid unnecessary surgery.

\section{CV \\ $\nabla$}

Gibran Yusuf is an interventional radiology fellow at King،s College Hospital with a special interest in ultrasound. He qualified from Guy's, King's and St Thomas' medical school in 2008 having also performed attachments in Barbados and St Lucia and an intercalated Batchelor of Science in psychology. He continued to complete his 
foundation training in the William Harvey Hospital, Kent and Canterbury Hospital and the Princess Royal Hospital. He then commenced radiology training at King's College Hospital in 2010 and immediately gained an interest in ultrasound and interventional radiology, in particular contrast enhanced ultrasound.

Whilst completing his FRCR examinations he has published in peer reviewed journals on several aspects of ultrasound particularly in relation to contrast-enhanced ultrasound. He has learnt under the tutelage of eminent professors in the field and now performs contrast enhanced ultrasound in his routine practice as well as within his interventional practice. He also reviews for peer review journals.

He has spoken at a number of national and international meetings as well as having published multiple conference abstracts. In addition he won the young investigator award for the British Medical Ultrasound Society in 2014 and continued his success by winning the European young investigator award at EUROSON 2015. He was also nominated for the trainee research prize at RSNA for his work on the safety and cost analysis of paediatric contrast enhanced ultrasound. His current research interests include the application of non-hepatic uses of contrast enhanced ultrasound, elastography and radiation dose reduction in children as well as non-invasive ultrasound in the assessment of liver disease. 